\title{
Integral dose investigation of non-coplanar treatment beam geometries in radiotherapy
}

\author{
Dan Nguyen and Peng Dong \\ Department of Radiation Oncology, University of California, Los Angeles, California 90095
}

Troy Long

Department of Industrial and Operations, University of Michigan, Ann Arbor, Michigan 48109

Dan Ruan and Daniel A. Low

Department of Radiation Oncology, University of California, Los Angeles, California 90095

Edwin Romeijn

Department of Industrial and Operations, University of Michigan, Ann Arbor, Michigan 48109

Ke Shenga)

Department of Radiation Oncology, University of California, Los Angeles, California 90095

(Received 18 June 2013; revised 19 November 2013; accepted for publication 20 November 2013; published 17 December 2013)

Purpose: Automated planning and delivery of non-coplanar plans such as $4 \pi$ radiotherapy involving a large number of fields have been developed to take advantage of the newly available automated couch and gantry on $\mathrm{C}$-arm gantry linacs. However, there is an increasing concern regarding the potential changes in the integral dose that needs to be investigated.

Methods: A digital torso phantom and 22 lung and liver stereotactic body radiation therapy (SBRT) patients were included in the study. The digital phantom was constructed as a water equivalent elliptical cylinder with a major axis length of $35.4 \mathrm{~cm}$ and minor axis of $23.6 \mathrm{~cm}$. A $4.5 \mathrm{~cm}$ diameter target was positioned at varying depths along the major axis. Integral doses from intensity modulated, noncoplanar beams forming a conical pattern were compared against the equally spaced coplanar beam plans. Integral dose dependence on the phantom geometry and the beam number was also quantified. For the patient plans, the non-coplanar and coplanar beams and fluences were optimized using a column generation and pricing approach and compared against clinical VMAT plans using two full (lung) or partial coplanar arcs (liver) entering at the side proximal to the tumor. Both the average dose to the normal tissue volume and the total volumes receiving greater than 2 Gy (V2) and 5 Gy (V5) were evaluated and compared.

Results: The ratio of integral dose from the non-coplanar and coplanar plans depended on the tumor depth for the phantom; for tumors shallower than $10 \mathrm{~cm}$, the non-coplanar integral doses were lower than coplanar integral doses for non-coplanar angles less than $60^{\circ}$. Similar patterns were observed in the patient plans. The smallest non-coplanar integral doses were observed for tumor $6-8 \mathrm{~cm}$ deep. For the phantom, the integral dose was independent of the number of beams, consistent with the liver SBRT patients but the lung SBRT patients showed slight increase in the integral dose when more beams were used. Larger tumor size and larger patient body size did not change the overall relationship of integral doses between non-coplanar and coplanar cases. However, the thin disk-shaped tumor received at least $40 \%$ greater integral doses with the non-coplanar plans. Overall, patient non-coplanar integral doses and V5 were comparable to those of coplanar doses from the same optimization engine and 15\%-20\% lower than state of the art VMAT plans. However, non-coplanar beams significantly increased V2 in both the phantom and patients. On average, the lung and liver SBRT patient normal tissue volumes receiving dose greater than 2 Gy were increased by 749 and $532 \mathrm{~cm}^{3}$, respectively.

Conclusions: The authors used a digital phantom simulating a patient torso and 22 SBRT patients to show that the integral doses from the plans employing optimized non-coplanar beams are comparable to those of the coplanar plans using an equal number of discrete beams and are significantly lower than those of VMAT plans. The non-coplanar beams expose a larger normal tissue volume to non-zero doses, whose impact will need to be evaluated individually to determine the risk/benefit ratio of the non-coplanar plans. () 2014 American Association of Physicists in Medicine. [http://dx.doi.org/10.1118/1.4845055]

Key words: non-coplanar, integral dose, intensity modulated 


\section{INTRODUCTION}

Non-coplanar beams provide additional angles for critical organ sparing but their utilization in external beam radiotherapy has been limited with the exception of specialized stereotactic radiosurgery (SRS) machines such as Gamma Knife and CyberKnife and conventional linear accelerator-based SRS using cone collimators and multiple intersecting arcs. In principle, the non-coplanar beam solution space is a superset of the coplanar beam solution space and when appropriately implemented should always improve nearby critical organ sparing. Technical challenges including collision hazards, potentially long delivery times, the lack of practical integrated beam orientation and fluence optimization tools, and subsequent evidence to support the clinical significance of non-coplanar planning have hampered the adoption of non-coplanar radiotherapy on the most widely available $\mathrm{C}$-arm gantry systems.

Notwithstanding, steady progress has been made in noncoplanar treatment planning and delivery research. On the optimization front, an intuitive approach to the beam orientation selection by minimizing the projected organs-at-risk (OAR) areas overlapping the projected planning target volume (PTV) has been developed by Haas et al. ${ }^{1}$ The method was developed further by Pugachev et al. ${ }^{2}$ by calculating the path-of-least-resistance (PoLR) using back projection methods. PoLR was computationally inexpensive but its geometrical objective function differed from dosimetric goals, requiring manual adjustment to resolve conflicts. It also decoupled the beam orientation selection processes from intensity modulation, potentially preventing it from achieving near-optimal solutions. Based on direct dose objectives, genetic, ${ }^{3,4}$ simulated annealing, ${ }^{5}$ nested partitions, ${ }^{6,7}$ and particle swarm ${ }^{8}$ algorithms have been used to solve the integrated beam orientation optimization and intensity modulation problem with varying degrees of success, suffering from common bottlenecks in computational efficiency and robustness. More recently, Breedveld et al. presented a multicriteria integrated beam angle and fluence optimization method, termed iCycle, ${ }^{9}$ to achieve clinical dosimetric objectives. It has been shown that the iCycle plans involving non-coplanar beams consistently outperformed coplanar plans in tumor coverage and critical organ sparing. ${ }^{10,11}$ We have developed an integrated beam orientation and fluence optimization method, termed $4 \pi$ radiotherapy, ${ }^{12-14}$ to address issues related to the preplanning modeling of the beam geometry solution space, defined as the range of available couch and gantry angles, that avoids collision. $4 \pi$ radiotherapy also addresses the computational performance of non-coplanar plans involving a large number of non-coplanar beams, which has been recognized as an essential element contributing to the dosimetric improvement. ${ }^{10,13}$ The optimization method is based on a column generation and pricing approach that instead of calculating the objective function explicitly for each additional beam or beamlet, it evaluates the first order information of the objective function to improve computational efficiency. ${ }^{15}$ Non-coplanar plans involving up to 30 beams were optimized within the common time frame allocated for IMRT plan generation. We showed that for liver and lung stereotactic body radiation therapy (SBRT), non-coplanar plan dose distributions were substantially more compact than the clinical plans and contained steeper dose gradients outside the PTV. The physical dose improvement could lead to clinically meaningful dose escalation without increasing critical organ doses. $4 \pi$ radiotherapy plans have been shown to be deliverable within 20 min on the widely available linacs equipped with computercontrolled gantries and couches.

These recent developments have revitalized interest in noncoplanar planning. While the optimization and delivery methods have been maturing to demonstrate the dosimetric improvements for various clinical sites, a remaining question to assess the use of non-coplanar beams is whether the large number of non-coplanar beams increases the integral dose which has been associated with the probability of developing secondary malignancies. ${ }^{16-18}$ Answers to this question will impact the cost-benefit analysis of non-coplanar beam plans. Here, we use both a digital phantom and patient data to quantify integral doses differences between coplanar and non-coplanar treatment plans.

\section{METHODS AND MATERIAL}

\section{A. Digital phantom study}

A digital water equivalent elliptical cylinder [Fig. 1(a)], with major and minor axes dimensions of 35.4 and $23.6 \mathrm{~cm}$, respectively, was constructed to simulate a relatively slim patient torso. The length of the phantom was sufficient to encompass all non-coplanar beam paths. A $4.5 \mathrm{~cm}$ diameter spherical target was positioned in five equal intervals along the major axis from the center to the edge. The volume of the target is $47.7 \mathrm{~cm}^{3}$. For each target position, coplanar and non-coplanar beam arrangements were employed. To maintain generality, no OARs were included in the phantom model. Because there was no critical organ to avoid, optimization of beam orientations resulted in orientations that do not resemble patient plans. Instead, for the non-coplanar case, 20 beams were chosen at angles and positions that formed a circular cone converging on the target [Figs. 1(a)-1(c)] and entering at the surface that is nearest to the target. The beam pattern was a geometric approximation of the liver SBRT $4 \pi$ radiotherapy beam geometry such as the patient shown in Fig. 1(d). ${ }^{12}$ The angle between entering beams and the cone axis of symmetry was labeled the cone angle. The cone axis position was selected to minimize the surface to target distance along the cone axis direction. Twenty equally spaced beams were also used in the coplanar geometry.

Four cone angles were studied: $24^{\circ}, 36^{\circ}, 48^{\circ}$, and $60^{\circ}$. In addition to the 20 beams used for these cone angle configurations, the integral dose dependence to the number of beams was evaluated for the $48^{\circ}$ angle using 10,20 , and 30 beams.

To study integral dose dependence on the phantom geometry, additional non-coplanar plans were created using the $36^{\circ}$ cone angle configuration and 20 beams. A second phantom with 51 and $34 \mathrm{~cm}$ along the major and minor axes was created to simulate a larger patient torso while keeping the same $4.5 \mathrm{~cm}$ spherical target. Since the original digital target 

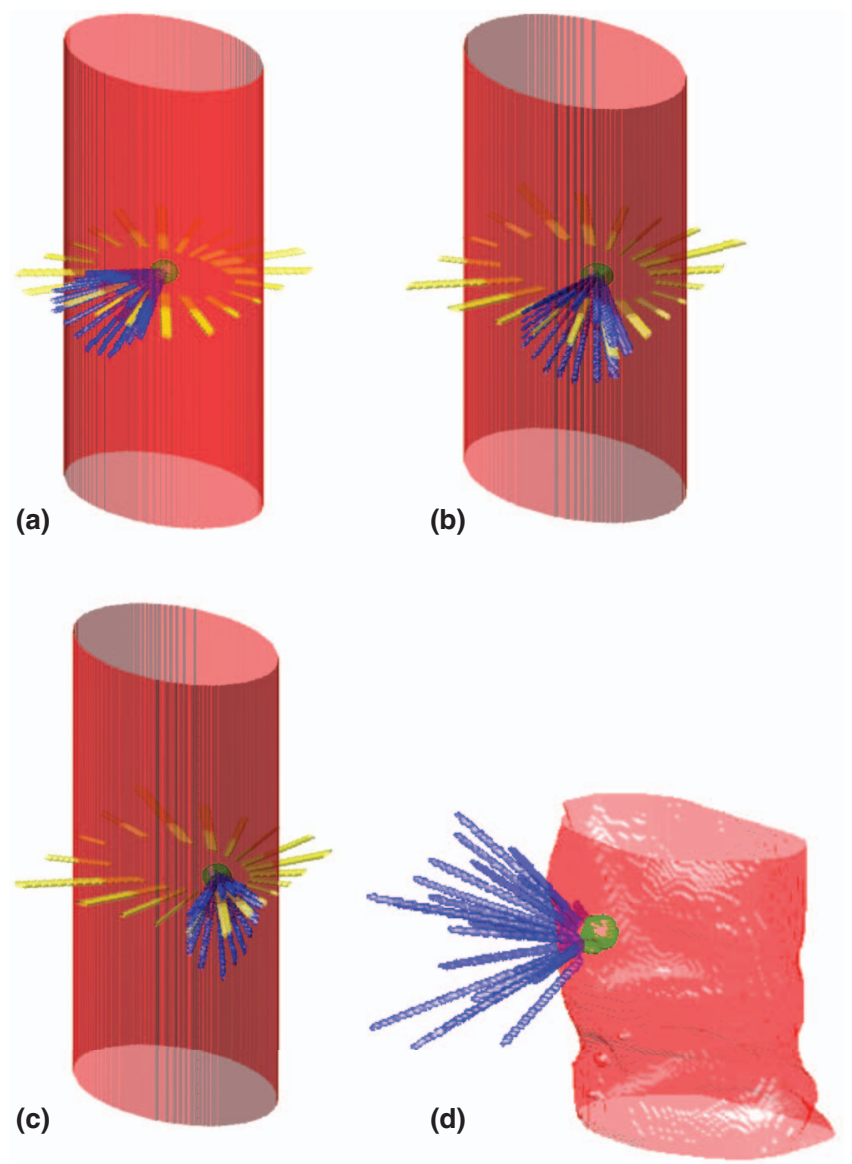

FIG. 1. 3D renderings of the elliptical cylindrical phantom with a target. (a)-(c) The pattern of non-coplanar beams to minimize surface to target distance as the target moves from center toward the edge along the major axis. (d) The optimized non-coplanar beam pattern for a typical liver cancer patient.

volume is on the lower end of SBRT PTV, a larger $5.9 \mathrm{~cm}$ diameter target model was added in the analysis. The larger spherical target volume is $107.5 \mathrm{~cm}^{3}$. A thin disk-shaped target that is $9 \mathrm{~mm}$ thick and $4.5 \mathrm{~cm}$ in diameter was also created. Both target variants were used in the smaller cylinder phantom. Both coplanar and non-coplanar beams were optimized to deliver a uniform $60 \mathrm{~Gy}$ dose to these targets.

\section{B. Patient study}

Twelve SBRT lung and 10 liver cases previously treated by IMRT or VMAT were planned using the $4 \pi$ method. The PTV volumes and depths are summarized in Table I. The prescription dose was defined as the dose received by $95 \%$ of the PTV volume. The PTV and critical organ doses were previously reported ${ }^{12,14}$ and we will focus primarily on the integral dose.

The planning process began by distributing 1162 noncoplanar candidate beams throughout the entire $4 \pi$ solid angle space with $6^{\circ}$ of separation between two nearest neighbor beam pairs. From the candidate pool, we eliminated those beams that would cause collisions between the gantry and the couch or patient as determined using a precise computer assisted design (CAD) models of the linear accelerator (Varian
TABLE I. PTV volume and depth (from nearest body surface to the PTV center-of-weight) of 10 liver and 12 lung patients.

\begin{tabular}{lcccc}
\hline \hline \multicolumn{2}{c}{ Liver patients } & & \multicolumn{2}{c}{ Lung patients } \\
\cline { 1 - 2 } \cline { 5 - 5 } PTV volume $\left(\mathrm{cm}^{3}\right)$ & PTV depth $(\mathrm{cm})$ & & PTV volume $\left(\mathrm{cm}^{3}\right)$ & PTV depth $(\mathrm{cm})$ \\
\hline 59.2 & 5.0 & & 117.0 & 7.5 \\
123.0 & 7.5 & & 138.4 & 5.2 \\
64.8 & 8.3 & & 43.6 & 5.9 \\
88.4 & 10.2 & & 9.6 & 9.7 \\
35.6 & 11.3 & & 138.5 & 9.0 \\
46.5 & 4.6 & & 50.7 & 7.3 \\
53.5 & 9.2 & & 135.0 & 5.5 \\
109.2 & 7.7 & & 30.6 & 6.2 \\
128.9 & 6.4 & & 80.3 & 7.0 \\
10.6 & 5.6 & & 100.3 & 5.1 \\
& & 105.7 & 11.0 \\
& & 70.0 & 6.2 \\
\hline \hline
\end{tabular}

EX) and a human subject and simulating their relative positions for each candidate beam. To generally increase the deliverability for larger patients and add a safety margin, extended focus-to-tumor-distances (FTD) are needed in $4 \pi$ radiotherapy but implementation of the concept is beyond the scope of the current study.

\section{C. Dose calculation and optimization}

For both the phantom and patient studies, the individual beams were subdivided into $6 \times 6 \mathrm{~mm}^{2}$ beamlets and the dose distribution matrices of each beamlet were calculated using an in-house collapsed-cone convolution/superposition code based on $6 \mathrm{MV} \mathrm{x}$-ray poly-energetic kernels, which were segmented into 24 concentric circles with varying radii from 0.1 to $60 \mathrm{~cm}$, and 48 zenithal segmentations equally spaced from $0^{\circ}$ to $180^{\circ}$. Heterogeneity corrections were applied in all plans. The dose calculation resolution was $3 \times 3 \times 3 \mathrm{~mm}^{3}$.

The algorithmic details and validation results of the optimization modeling was previously introduced. ${ }^{12,14,15}$

The beam orientation optimization (BOO) and fluence map optimization (FMO) were performed interleaved very efficiently using CPLEX (Academic Research Edition 12.2). Beams were added in an iterative process until the desired number of beams was reached, which were 22 and 30 beams for the liver and lung plans, respectively. As mentioned BOO was not performed on the phantoms. Instead, fixed conical beams typically observed in patient $4 \pi$ plans were used. Integral dose was calculated as the average dose received by the entire volume excluding PTV. The volumes receiving greater than 2 Gy (V2) and 5 Gy (V5) were also calculated.

Two-arc volumetric modulated arc therapy (VMAT) plans were created as the reference clinical plans on Eclipse (Varian, Palo Alto, CA). The dose calculation accuracy was 2.5 $\times 2.5 \times 2.5 \mathrm{~mm}^{3}$; anisotropic analytical algorithm (AAA) and heterogeneity corrections were applied. 

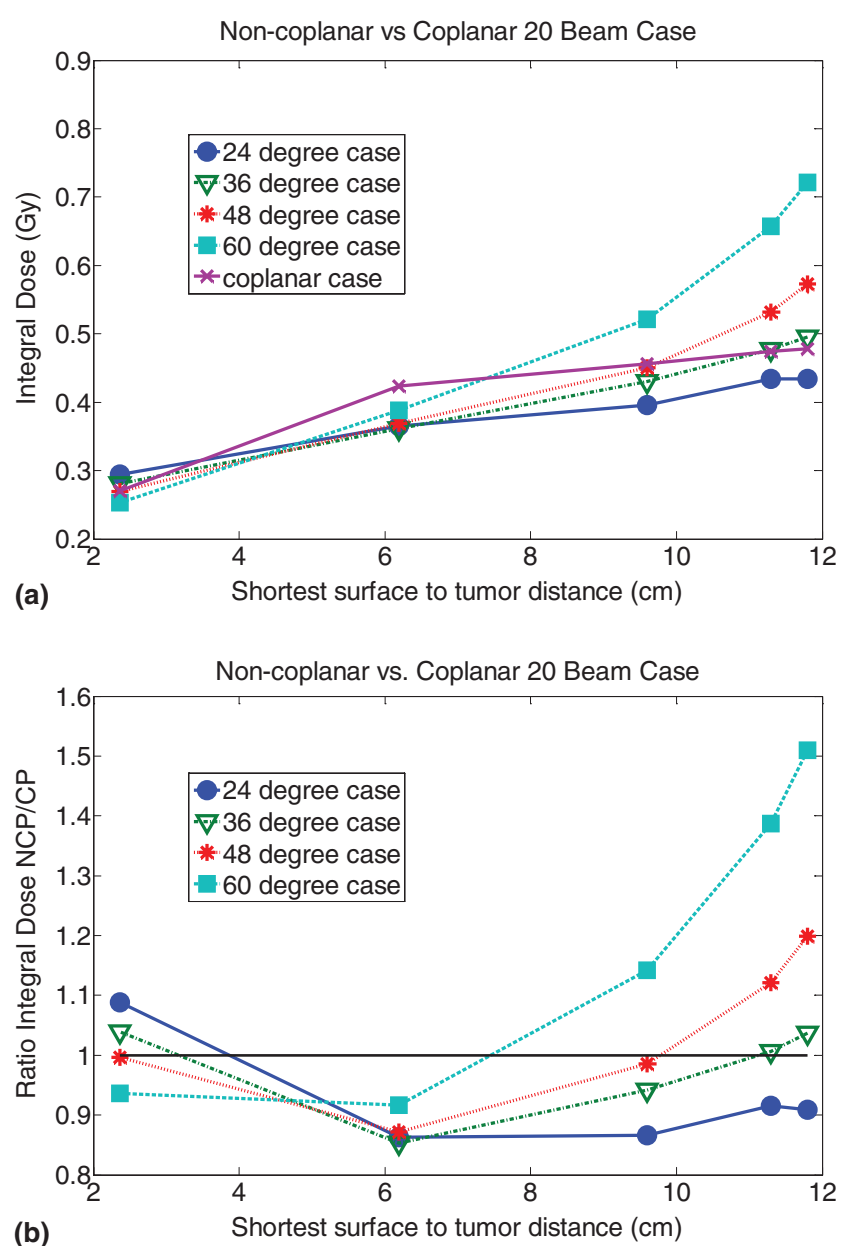

FIG. 2. Comparison of integral dose between coplanar and non-coplanar cases at different cone angles. (a) Integral dose vs tumor depths. (b) The ratios between non-coplanar and coplanar integral doses.

\section{RESULTS}

Figure 2 shows the phantom integral dose comparison between the coplanar and non-coplanar plans for varying digital target centroid depths. For all plans, integral dose increased with deeper target depths. Interestingly, the non-coplanar plan integral dose was greater than that of the coplanar plans at $2.3 \mathrm{~cm}$ depth, less than the coplanar plans at moderate depths and then again greater than the coplanar plans at deeper depths. This made the ratio of the non-coplanar to coplanar plan integral dose have the shape of a $\mathrm{U}$ as a function of depth with a maximal reduction of the integral dose between 6 and $8 \mathrm{~cm}$ depths (Fig. 2).

Figure 3 shows the integral dose between non-coplanar and coplanar plans for the $48^{\circ}$ cone angle with varying depth and as a function of the number of beams. The integral doses did not vary substantially with the number of beams. The volumes receiving greater than 2 or $5 \mathrm{~Gy}$ are shown in Fig. 4. With the exception of the most narrow cone angle of $24^{\circ}$, non-coplanar beams increased V2 but not V5.

Figure 5 shows non-coplanar to coplanar integral dose ratios for the three variants of the model. The ratio between noncoplanar and coplanar integral doses decreased when larger

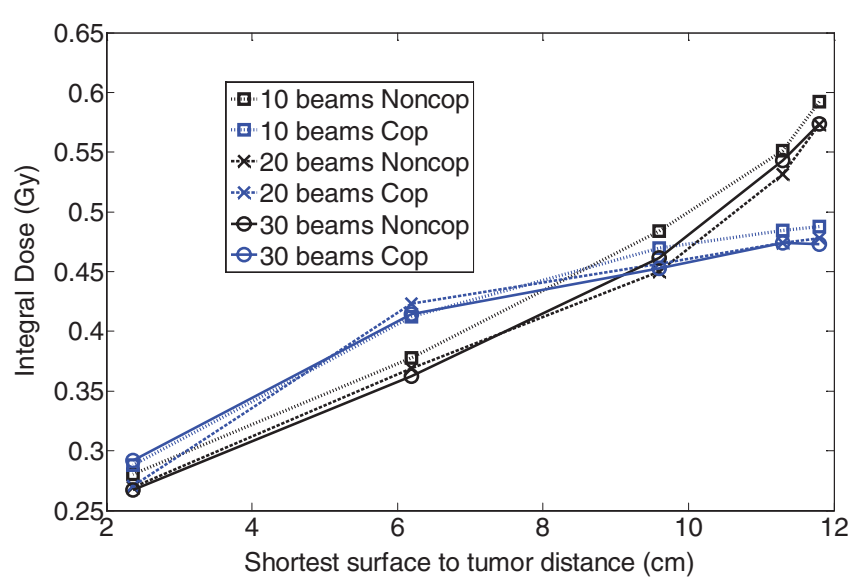

FIG. 3. Integral dose ratios between the non-coplanar and coplanar plans using 10,20 , and 30 beams.

phantom increased with larger targets. Both changes were relatively small and maintained the $\mathrm{U}$ shaped pattern. The thin disk-shaped target model resulted in significantly greater integral dose with the non-coplanar plans than with the coplanar plans. Therefore, caution should be exercised when planning for targets of these shapes. However, it should be noted, that the thin disk-shaped PTV is rare in reality due to both
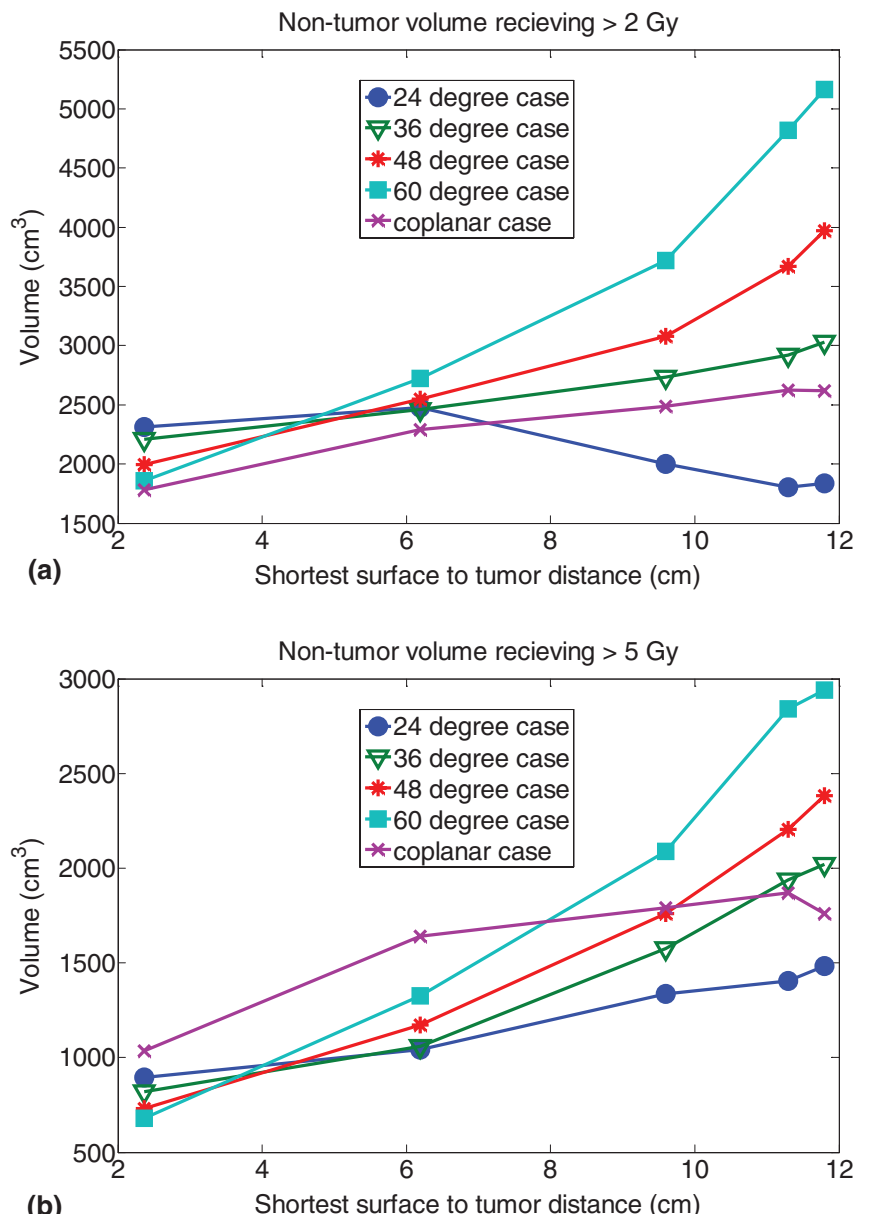

(b)

FIG. 4. The volumes receiving greater than (a) 2 Gy or (b) 5 Gy vs noncoplanar beam cone angles for the phantom. 


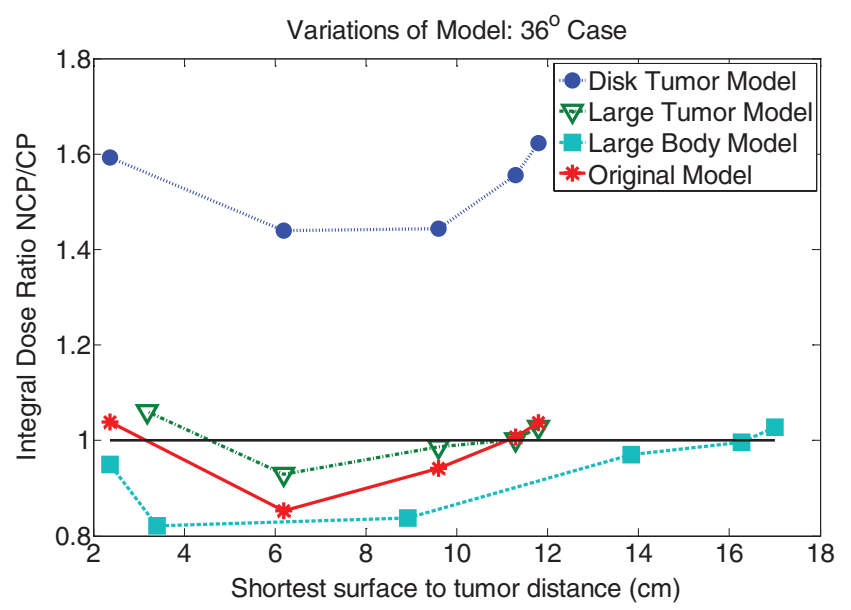

FIG. 5. Non-coplanar to coplanar integral dose ratios for the three different phantom variants: thin disk target, large spherical target, and large phantom torso.

tumor natural growth pattern and the additional margins that inevitably increase the thickness of target.

The average integral dose of lung non-coplanar plans was slightly less than that of coplanar plans (1.98 vs $2.00 \mathrm{~Gy}$, p $=0.97$ ). Both were significantly less than the average integral dose for clinical VMAT plans (2.49 Gy, $\mathrm{p}=0.01)$. For liver cases, the average integral dose for non-coplanar plans was slightly greater than coplanar plans (1.37 vs $1.33 \mathrm{~Gy}, \mathrm{p}$ $=0.19)$. Both were significantly less than the VMAT plans (1.63 Gy, $\mathrm{p}=0.002)$. The semilog cumulative dose volume histograms of the phantom and a liver SBRT patient show that the non-coplanar plan exposed a smaller volume to 5-50 Gy and a larger volume to less than 5 Gy (Fig. 6).

For the lung SBRT patients, a similar U-shaped pattern was demonstrated that the integral dose ratios between noncoplanar and coplanar plans decreased first and then increased. The minimal ratio was observed at $6-8 \mathrm{~cm}$ tumor depths. The liver data show a similar but noisier dependence pattern on the tumor depths (Fig. 7).

For the lung SBRT cases, the integral dose increased slightly with more beams but no trend was clear for the liver SBRT cases. V5 of the non-coplanar and coplanar plans were close but the V2 of non-coplanar plans were significantly greater as shown in Fig. 8. The lung and liver SBRT patient normal tissue volumes receiving dose greater than $2 \mathrm{~Gy}$ were on average increased 749 and $532 \mathrm{~cm}^{3}$.

The target dose homogeneities are different in the phantom and the patient plans. In the phantom plans, a uniform prescription dose was used and achieved. In the patient plans, hot spots up to $20 \%$ of the prescription doses were allowed. The actual average maximum doses were $109.8 \%$ and $119.0 \%$ for the liver plan and the lung plans, respectively. We were not able to generate dose hot spots greater than $110 \%$ of the prescription dose in the phantom plans despite removing the dose uniformity constraint. This was because hot spots with greater doses did not improve the objective function. However, for these phantom plans with more heterogeneous doses, both the coplanar and non-coplanar integral doses were
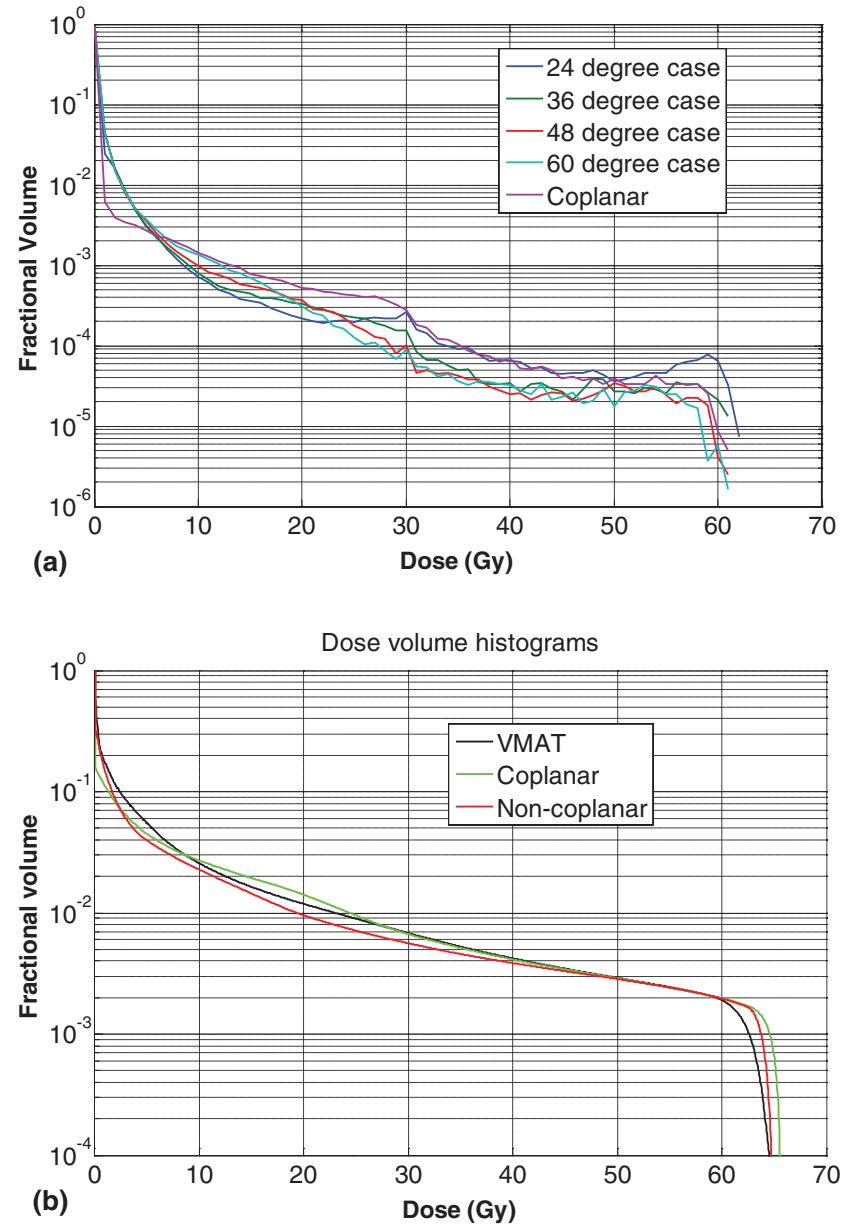

FIG. 6. Semilog differential dose volume histogram of the phantom (a) and a typical liver patient (b).

slightly reduced resulting in a small net shift in the noncoplanar/coplanar integral dose ratios as shown in Fig. 9. Therefore, target dose heterogeneity did not appear to influence our conclusion.

\section{DISCUSSION}

Integral dose and the normal tissue volume receiving low radiation doses are two important parameters to evaluate the delivery efficiency of a planning system. Ideally, minimization of the integral dose indicates an optimal physical

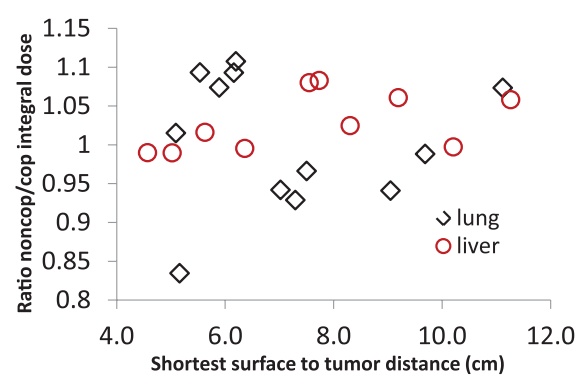

FIG. 7. The ratio between non-coplanar and coplanar integral doses vs the tumor depth for lung and liver patients. 
(a) Liver case integral dose

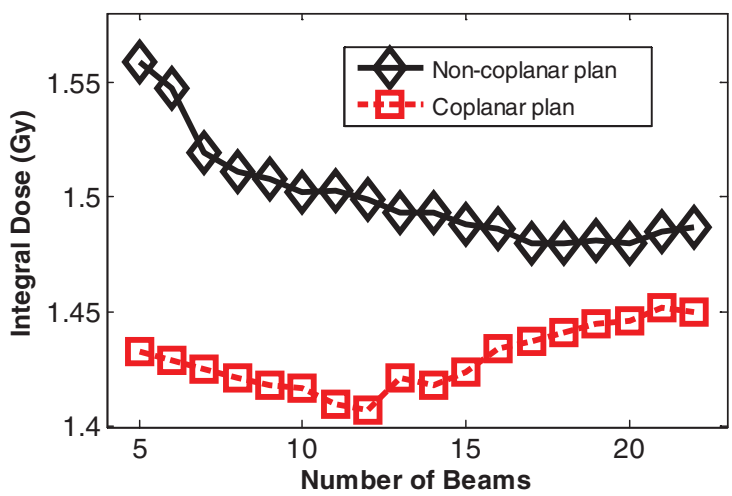

(c) lung case integral dose

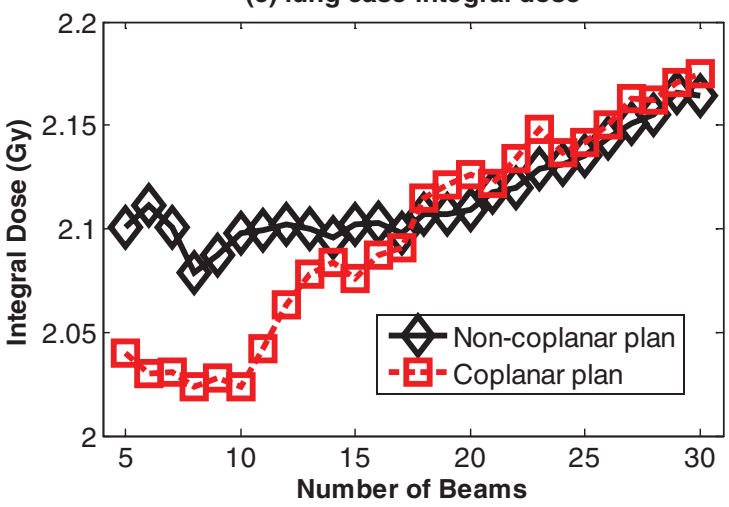

(b) Liver case dose volume

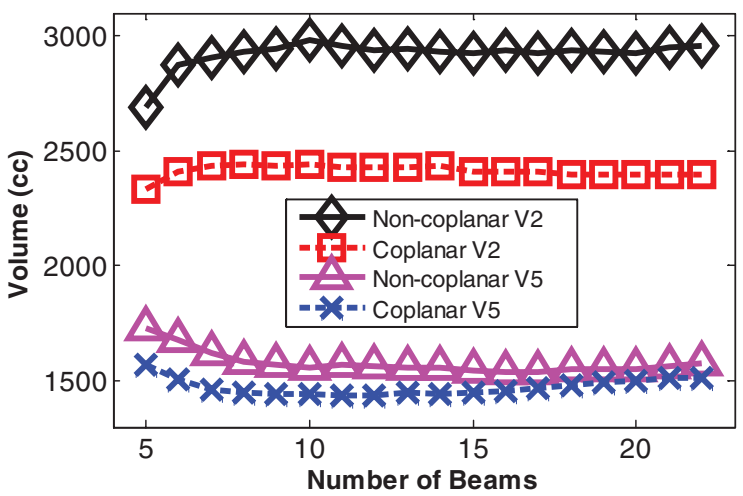

(d) lung case dose volume

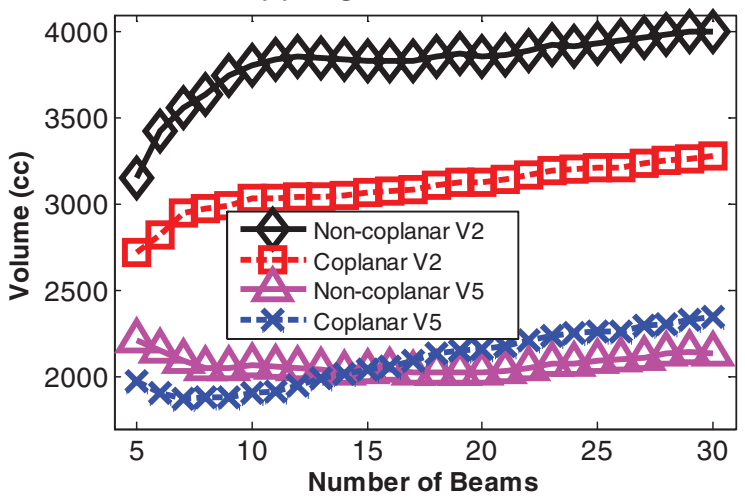

FIG. 8. The integral dose of and volumes receiving greater than 2 or $5 \mathrm{~Gy}$ vs non-coplanar beam cone angles for the liver (a-b) and lung (c-d) patients.

efficiency of a given treatment platform. On the other hand, integral dose is not specific and quite often dominated by large volumes of less critical or less radiosensitive tissues. It has been shown that $97 \%$ of the radiation energy was delivered to the normal tissue in a typical coplanar prostate plan, regardless of the number of beams used. ${ }^{19}$ Furthermore, the adverse effect of low dose spread is stochastic and overall low probability events. Therefore, in clinical practice, integral dose is not commonly evaluated as part of the quality

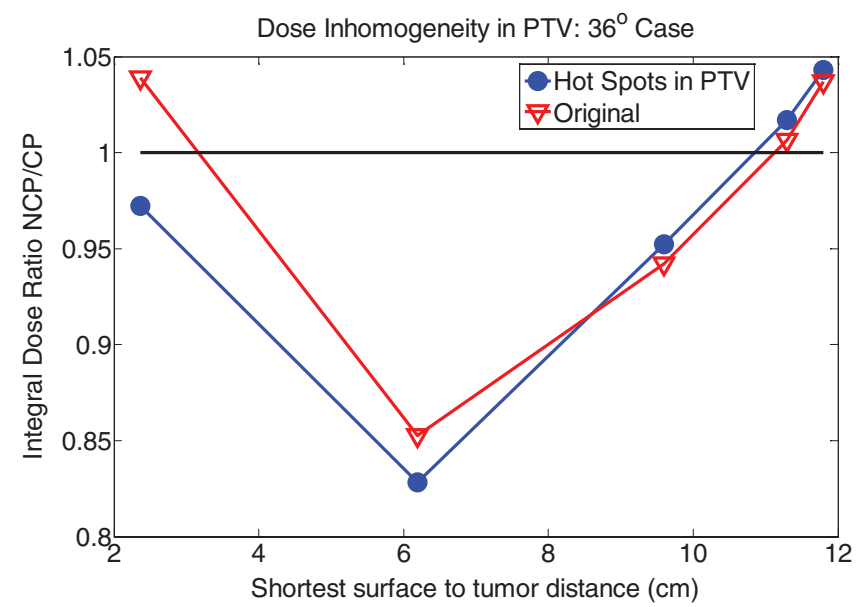

FIG. 9. The integral dose ratios between non-coplanar and coplanar plans with and without dose homogeneity constraints. indices and higher priorities are given to more urgent needs in tumor control and deterministic normal organ toxicity from high dose exposure. With that in mind, quantification of the integral dose is important when there is a potential paradigm shift from the coplanar-dominant planning method to the noncoplanar planning platform. Although integral dose was found to be relatively constant for a given delivery geometry and insensitive to differences in intensity modulation, ${ }^{19,20}$ the platform change may result in a substantial change in the integral dose.

In the study, we showed that, with the exception of the thin disk tumor case, the integral dose can actually be lowered or kept at the same level with extensive use of non-coplanar beams. The somewhat counterintuitive and nonlinear behavior of the integral dose is due to intensity modulation and the nonlinear percent depth dose curve of $6 \mathrm{MV}$ x-rays. A similar tumor depth dependence pattern was observed in the patients with greater fluctuation attributing to: (1) the patient physical tumor depths are different from the radiological depths; (2) the beam orientations were selected by the optimization engine and different from the equally distributed conical pattern and coplanar beams used in the phantom study; and (3) individual variation in anatomy. Nonetheless, the 22patient-study showed insignificant changes in integral dose when an automated beam orientation optimization program was used to select discrete coplanar and non-coplanar beam angles. More importantly, regardless of the coplanarity, the discrete beam plans consistently resulted in statistically sig- 
nificantly lower integral dose compared against state of the art VMAT plans. The observation agreed with previous dosimetric studies showing that rotational methods, including VMAT (Ref. 21) and TomoTherapy, ${ }^{22}$ delivered $15 \%$ greater integral dose than IMRT employing discrete beams. Therefore, this study should relieve the concerns about the integral dose of highly non-coplanar $4 \pi$ plans.

On the other hand, the volume exposed to very low doses was increased by use of non-coplanar beams as shown by the study. The tradeoff was analogous to the transition from simple parallel-opposed field treatments to multiple-field IMRT and more recently rotational IMRT. The potential risk embedded in the previous transition was characterized by Hall and Wuu. ${ }^{18}$ The increased low dose volume could increase the secondary cancer occurrence rates for patients having survived their original cancers. Similarly, for highly noncoplanar radiotherapy, a tradeoff was found between significantly more compact dose distributions ${ }^{12,14}$ and the additional normal tissue volume receiving low doses and the associated long-term stochastic effect. For example, the significance of V2 depends on the patient's clinical conditions and should be balanced against treatment plan dosimetric benefits.

The study is based on a digital phantom simulating patient torso and body radiotherapy. For patients with cancer located in the head and neck, extremities and very peripheral organs such as the breast, the analysis may not apply. We will establish non-coplanar beam patterns for these sites and revisit the topic for better understanding of the integral dose.

We only studied the integral doses of 6 MV x-rays, which are dominantly used in IMRT, and more so in the rotational IMRT plans for comparison in this project. Higher or lower $\mathrm{x}$ ray energies may result in relative shift of the positions in the comparison. It has been reported that for deep-seated targets and coplanar plans, the integral dose is nearly independent of $\mathrm{x}$-ray energy for 6,10 , and $18 \mathrm{MV}$ x-rays. ${ }^{23}$ In comparison, the non-coplanar plan integral dose would likely increase for higher x-ray energies, whose deeper penetration would have a greater combined effect with the longer beam pathlengths of non-coplanar beams. To accurately assess the changes in integral doses, these higher energy beams need to be modeled in a non-coplanar planning program.

In the patient study, we choose to compare $4 \pi$ to VMAT so the integral dose can be referenced to a clinical system. A potential issue with this comparison us that the integral dose could be algorithm-dependent. To minimize the errors, we have tuned our dose calculation model to match the $6 \mathrm{MV}$ measurement data of a Varian machine, which was also used to commission the Eclipse planning system. Furthermore, as explained in the method and material, we fully sampled the dose deposition kernel for all beamlet calculation to prevent underestimating scatter doses. Therefore, the difference between calculation platforms should be small and not affect the conclusion.

\section{CONCLUSION}

Integral dose dependence on plan coplanarity, tumor depths, and beam number was studied. For the phantom, the integral dose of non-coplanar plans was lower than coplanar plans within a specific depth range that covers a wide range of clinical cases. The conclusion was only insignificantly influenced by the phantom and tumor sizes. However, for thin disk-shaped tumor, the non-coplanar plan integral dose was over $40 \%$ greater, requiring particular caution for these cases. For patients with more complex geometry and optimization objective functions, non-coplanar plans did not significantly increase the integral dose while achieving superior tumor coverage and critical organ sparing. However, the normal tissue volume receiving low dose was increased with non-coplanar beams. The clinical significance of the increase could depend on patient clinical condition and should be individually evaluated.

a) Author to whom correspondence should be addressed. Electronic mail: ksheng@mednet.ucla.edu

${ }^{1}$ O. C. Haas, K. J. Burnham, and J. A. Mills, "Optimization of beam orientation in radiotherapy using planar geometry," Phys Med. Biol. 43, 21792193 (1998).

${ }^{2}$ A. B. Pugachev, A. L. Boyer, and L. Xing, "Beam orientation optimization in intensity-modulated radiation treatment planning," Med. Phys. 27, 1238-1245 (2000).

${ }^{3}$ Q. Hou, J. Wang, Y. Chen, and J. M. Galvin, "Beam orientation optimization for IMRT by a hybrid method of the genetic algorithm and the simulated dynamics," Med. Phys. 30, 2360-2367 (2003).

${ }^{4}$ D. P. Nazareth, S. Brunner, M. D. Jones, H. K. Malhotra, and M. Bakhtiari, "Optimization of beam angles for intensity modulated radiation therapy treatment planning using genetic algorithm on a distributed computing platform," J. Med. Phys. 34, 129-132 (2009).

${ }^{5} \mathrm{~J}$. Stein et al., "Number and orientations of beams in intensity-modulated radiation treatments," Med. Phys. 24, 149-160 (1997).

${ }^{6}$ W. D. D’Souza, H. H. Zhang, D. P. Nazareth, L. Shi, and R. R. Meyer, "A nested partitions framework for beam angle optimization in intensity-modulated radiation therapy," Phys. Med. Biol. 53, 3293-3307 (2008).

${ }^{7}$ X. Jia, C. H. Men, Y. F. Lou, and S. B. Jiang, "Beam orientation optimization for intensity modulated radiation therapy using adaptive $1(2,1)$ minimization,” Phys. Med. Biol. 56, 6205-6222 (2011).

${ }^{8}$ Y. J. Li, D. Z. Yao, J. Yao, and W. F. Chen, "A particle swarm optimization algorithm for beam angle selection in intensity-modulated radiotherapy planning," Phys. Med. Biol. 50, 3491-3514 (2005).

${ }^{9}$ S. Breedveld, P. R. Storchi, P. W. Voet, and B. J. Heijmen, "iCycle: Integrated, multicriterial beam angle, and profile optimization for generation of coplanar and noncoplanar IMRT plans," Med. Phys. 39, 951-963 (2012).

${ }^{10}$ L. Rossi, S. Breedveld, B. J. Heijmen, P. W. Voet, N. Lanconelli, and S. Aluwini, "On the beam direction search space in computerized noncoplanar beam angle optimization for IMRT-prostate SBRT," Phys. Med. Biol. 57, 5441-5458 (2012).

${ }^{11}$ P. W. Voet, S. Breedveld, M. L. Dirkx, P. C. Levendag, and B. J. Heijmen, "Integrated multicriterial optimization of beam angles and intensity profiles for coplanar and noncoplanar head and neck IMRT and implications for VMAT," Med. Phys. 39, 4858-4865 (2012).

${ }^{12}$ P. Dong et al., " $4 \pi$ non-coplanar liver SBRT: a novel delivery technique," Int. J. Radiat. Oncol., Biol., Phys. 85, 1360-1366 (2013).

${ }^{13}$ P. Dong et al., " $4 \pi$ noncoplanar stereotactic body radiation therapy for centrally located or larger lung tumors," Int. J. Radiat. Oncol., Biol., Phys. 86, 407-413 (2013).

${ }^{14} \mathrm{P}$. Dong et al., " $4 \pi$ noncoplanar stereotactic body radiation therapy for centrally located or larger lung tumors," Int. J. Radiat. Oncol., Biol., Phys. 86(3), 407-413 (2013).

${ }^{15}$ H. E. Romeijn, R. K. Ahuja, J. F. Dempsey, and A. Kumar, "A column generation approach to radiation therapy treatment planning using aperture modulation," SIAM J. Optim. 15, 838-862 (2005).

${ }^{16}$ H. Engels, H. G. Menzel, P. Pihet, and A. Wambersie, "Risk assessment for cancer induction after low- and high-LET therapeutic irradiation," Strahlenther. Onkol. 175(Suppl 2), 47-51 (1999). 
${ }^{17}$ M. D'Arienzo, S. G. Masciullo, V. de Sanctis, M. F. Osti, L. Chiacchiararelli, and R. M. Enrici, "Integral dose and radiation-induced secondary malignancies: comparison between stereotactic body radiation therapy and three-dimensional conformal radiotherapy," Int. J. Environ. Res. Public Health 9, 4223-4240 (2012).

${ }^{18}$ E. J. Hall and C. S. Wuu, "Radiation-induced second cancers: the impact of 3D-CRT and IMRT," Int. J. Radiat. Oncol., Biol., Phys. 56, 83-88 (2003).

${ }^{19}$ W. D. D'Souza II and I. I. Rosen, "Nontumor integral dose variation in conventional radiotherapy treatment planning," Med. Phys. 30, 2065-2071 (2003).

${ }^{20}$ A. S. Reese, S. K. Das, C. Curie, and L. B. Marks, "Integral dose conservation in radiotherapy," Med. Phys. 36, 734-740 (2009).
${ }^{21}$ S. Myrehaug et al., "A treatment planning and acute toxicity comparison of two pelvic nodal volume delineation techniques and delivery comparison of intensity-modulated radiotherapy versus volumetric modulated arc therapy for hypofractionated high-risk prostate cancer radiotherapy," Int. J. Radiat. Oncol., Biol., Phys., 82, e657-e662 (2012).

${ }^{22} \mathrm{C}$. Song et al., "Superiority of conventional intensity-modulated radiotherapy over helical tomotherapy in locally advanced non-small cell lung cancer. A comparative plan analysis," Strahlenther. Onkol. 188, 901-909 (2012).

${ }^{23}$ A. Pirzkall, M. P. Carol, B. Pickett, P. Xia, M. Roach 3rd, and L. J. Verhey, "The effect of beam energy and number of fields on photon-based IMRT for deep-seated targets,” Int. J. Radiat. Oncol., Biol., Phys. 53, 434-442 (2002). 